\title{
Engaging Students with a Mobile Game-Based Learning System in University Education
}

\author{
http://dx.doi.org/10.3991/ijim.v8i4.3991 \\ A. Bartel and G. Hagel \\ Kempten University of Applied Sciences, Germany
}

\begin{abstract}
In this contribution we present a game-based learning concept which is based on mobile devices. It focuses a joyful stabilization of knowledge and the engagement of students using the Gamification approach and its game mechanics. Previous findings how to promote students' motivation are adapted in the mobile context and discussed. A pre-evaluation of the prototype is described with its findings.
\end{abstract}

Index Terms-Game-Based Learning; Gamification; Mobile Learning; Motivation; University Education

\section{INTRODUCTION}

Due to the mobile diffusion, learning is not a matter of a specific time of occurrence or a particular location any more [1]. This fact offers possibilities to revolutionize the way education is by now. To use these possibilities successfully for teaching, combining mobile learning and game-based learning approaches with Gamification seems a proper chance. This is what Extended Mobile Gaming Education (eMgage) is about. As a quiz-based mobile learning application it is extended with game mechanics to increase students' motivation and engagement, possibly some of the most important prerequisites for learning [2].

\section{CONCEPT}

We had the vision to build a system which motivates students to stabilize and extend their knowledge in a sustainable and joyful way with the use of recent mobile technology. After tailoring an agile software engineering process to break down and operationalize these thoughts into appropriate aims as well as prioritizing them, we came to the following system architecture as a result of the requirements analyses.

\section{A. Architectural view}

The system consists of three parts, which interact together using the internet or a local intranet (see Fig. 1.). The system components are loose coupled and use a request-response model for their interaction. Due to the fact that we wanted the system components not only to communicate using plain text commands, we had to define a very flexible and extendible protocol. It is based on TCP/IP and the encoding and decoding of the communication is done manually. This provides the freedom in message contents as we use plain bytestreams for communication. In addition, possible problems with (un-) marshalling [3] which can bring along non-expected amounts of data, can be ignored. This makes the communication among the system components clearly arranged and fast.

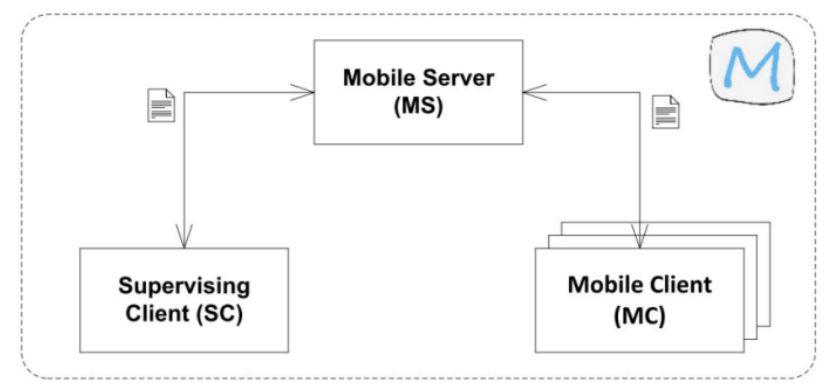

Figure 1. Overview of the learning system and relevant components.

Each system component follows a defined multilayered architecture [4]. Accordingly for example the infrastructure layer which the communication functionality is assigned to can be found in the three system components likewise. The functionalities of each system component is described in the following in more detail.

\section{B. Features}

The mobile server (MS) is responsible for providing data to the clients and channeling the communication and interaction between them. It accesses a MySQL database using a persistence layer which integrates the O/R mapping framework OrmLite [5]. It owns the business logic of the whole system. The supervising client (SC), a standalone Java client, controls the server, its data and provides instant feedback on mobile clients connected to the mobile server and their actions. The learning application eMgage is a mobile application client (MC), based on Android, which is used by students during university lectures or in non-university contexts (e.g. at home). It is constructed and build as a thin client since it primarily acts a presentation tier.

The application provides the following main features (see Fig. 2 main menu):

- Learnsessions capsulate different types of questions as a learning unit to a specific topic which can be edited by students.

- Personal profile to inform students about the recent achievements and knowledge state (see Fig. 2 profile page).

- Leaderboard to compare and compete with other participants.

- News area to inform students about upcoming events and learnsessions.

- Question builder to send in new suggestions for questions from students to the lecturer. 


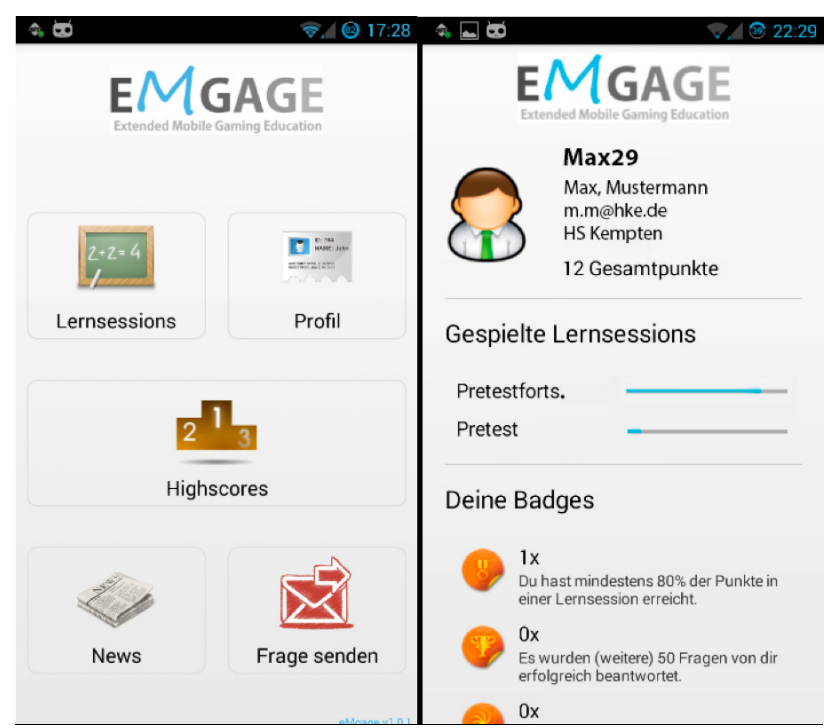

Figure 2. Screenshot main menu (left); overview profile page (right)

\section{Supporting the individual learning process}

In order to create sustainability in learning, student performances and related benchmarks have to be integrated in a broader process. As a temporal limitation of the process, the beginning and the end of a university course can be applied. The learning application eMgage offers the possibility to support this process by providing volunteer benchmarks on knowledge units as milestones of the process, which - not depending on the grade of success gives students feedback on their knowledge. Each milestone is connected with the processing of a learnsession by each student, either during lecture or in non-university contexts. Learnsessions deal with a defined topic, for example nonfunctional requirements in the context of software engineering.

They contain different types of questions (single choice, multiple choice and textual input) as well as their related answers. Each question is rated with a score and can be supported with different types of media (pictures, video streams and podcasts).

Students have different types of feedback tools when using eMgage. They derive from the Gamification approach and are summarized under the term "game mechanics" [6].

\section{The use of game mechanics in eMgage}

Gamification is defined as the concept of applying game-design thinking into none-game applications to make them more fun, engaging and change user's behavior. Game mechanics are "the mechanics of a gamified system which are made up of a series of tools, that when used correctly, promise to yield a meaningful response from the players" [6, p. 36]. Each feature of eMgage is connected to at least one game mechanic. The learning application integrates the following game mechanics: point systems, leaderboards and badges.

Point systems are described as an "absolute requirement for all gamified systems" [6, pp. 36]. According to the classification in [6] the applied point system in eMgage is based on experience points. This type provides the possibility to observe a learner, benchmark and lead him out of the view of a lecturer. Lecturers could observe the understanding of topics due to the average scores achieved in

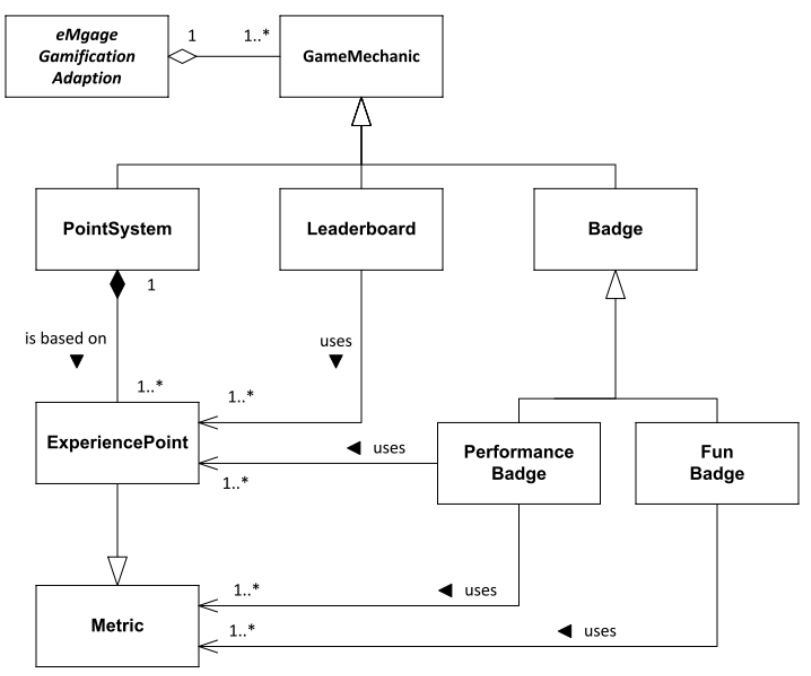

Figure 3. Meta model of game mechanics in eMgage

the related learnsessions. They can react respectively this average score for example by revisiting a topic with a different learning method. A comparable way of instant feedback is already successfully implemented in other learning scenarios like JiTT (Just-in-Time Teaching) [7, $8]$.

Experience points are expressed and compared in leaderboards. The type of leaderboard implemented in eMgage is described as local (limited to a university course) and non-infinite (limited to the users who participate in that course) [9]. It is used to increase the peer-group pressure in order to let students compete with each other. Due to the fact that every student is rated with the same metric system, performances are comparable. In case a participant recognizes a deficit in a topic he is able to appeal to another participant with a higher score, referring to the leaderboard. That is why leaderboards are often combined with a known and social environment [9].

Badges can be found in everyday life: sticky on the back of a car, as an award on clothes or as a virtual rating on e-commerce platforms like eBay. Besides the aim to signal status and social standing, badges are often used to control or guide a progress [9]. In eMgage there are two different types of badges: performance badges and fun badges. As the term indicates, performance badges are related to student performances hence to a specific metric. In order to achieve both seriousness and fun, special fun badges are implemented. They also use a metric for awarding but are primarily focused on non-performance achievements. An example awarding of a fun badge is the submitting of a suggestion for a question.

Fig. 3 summarizes the use of game mechanics in eMgage in a meta model using a UML class diagram. It indicates, that defining a particular metric each game mechanic is an important part of the design [9].

Additionally to the features the learning application offers, there are some more areas of impact which can positively influence students' interest in a topic and their learning motivation.

\section{Motivational AREAS OF IMPACT}

Learning motivation itself is hard to foster and describes the willingness of students to address sensory, cognitive and motoric capacities in a predefined learning 


\section{SHORT PAPER \\ Engaging Students With a Mobile GAME-BASEd LEARning SySTEM IN UNIVERSity EdUCATION}

situation and to coordinate in a way suitable for achieving a clearly defined objective [2]. However, we found four areas to be very effective in increasing students' motivation and their interest in a topic: arouse curiosity, promote independence, adapt difficulty and create incentives [2]. These can be adapted in the mobile context.

\section{A. Arouse curiosity}

A study of the University of Hampshire has shown that more than 70 percent of the students participating in a course use their smartphone more than three times during a course [10]. These findings might not be representative for every university but indicate a high significance of mobile devices for students and their interest in this technology. Hence, most students are familiarized with this medium. In addition human beings have got a natural and "objective interest for everything new" [11, p. 229]. The familiarization with this medium and our natural interest are used to arouse students' curiosity. This is the reason why eMgage is based on mobile devices.

\section{B. Promote independence}

Learning processes are personalized and can differ in time (when to learn), place (where to learn) and context (under which circumstances) [12, pp. 18]. Due to the use of mobile devices these constraints are almost compensated and provide learners increasing independence to structure their personal learning process. In addition, the access to learning material and its sharing gets more comfortable and context specific information is provided just in time. The learning application eMgage profits from these aspects as it uses recent mobile technology.

\section{Adapt difficulty}

"Learners will only have a joyful learning and develop motivation when they consider themselves to be efficient" [2, p.1303]. An imperative prerequisite to achieve this state is to have a flexible level of learning. It is characterized by the parameters that transfer of learning and level of learning are adequate but still there is something new to discover for learners [2, p. 1304]. The three parameters cannot be ideal provided for each type of learner, but different forming can at least support plenty of different learner types. Therefore eMgage uses a simple but effective algorithm to rate each question on the basis of these parameters (lectures rate on their personal estimation):

- Evaluate each parameter with a value in the range from 1, which means low, to 3, what expresses high.

- Build the arithmetic mean of the three values.

- Ceil the whole arithmetic expression.

- Compute.

The result of this calculation is the score that can be achieved when the question is answered correctly by a student. Certainly there are additional parameters and aspects which have to be taken into account when constructing a learning exercise as described in [13]. In this case the main focus of attention should not be the overall competence-oriented construction of learning exercises, rather the learner-centric and structured sensitization what knowledge can be expected from learners.

\section{Create incentives}

Incentives like prices or rewards can be used to increase students' short-term learning motivation [2]. Most of them are limited to the "action-reaction-principle" and are not embedded in a broader process. An example could be the evaluation of a student's performance during semester by a lecturer where the action is the performance itself and the evaluation result is the reaction in an educational short-term context. A challenge here is to keep students learning motivation high in long-term situations beyond finished student performances. Therefore it is necessary to create sustainability which is not related to knowledge of students in the first place, rather addresses their learning motivation. That is related to the step before learning: promoting the interest in a topic by creating incentives when dealing with it.

\section{LIMITS AND CHALLENGES}

Game mechanics derived from the Gamification concept as well as our findings offer possibilities to promote the engagement of students. Actually there are some limitations which have to be taken into account when transferring these approaches into university education.

One of the biggest challenges is to combine these approaches in a way that the learning motivation of students does not only depend on the awards which can be achieved. This would imply that an existing intrinsic motivation shifts to a one-sided extrinsic motivation which is not driven by curiosity anymore [14]. In addition a reward approach can suffer under the circumstance that achieved awards do not satisfy students anymore when using them over a long period. Thus incentives have to be dynamic and steadily improved or changed, otherwise the learning motivation is in danger to decrease.

Another important aspect is the kind of person a university is dealing with. Students cannot be compared with each other across-the-board in their personalities. Each student personality is individual and the motivation to use and play with such an application can differ widely. Consequently competing with other students can work well or will just have no effect on some individuals [15]. Nonetheless many researchers support the thesis, that mobile learning can actually revolutionize learning issues: "Mobile devices really make a difference in education. Their possibilities allow defining new kinds of scenarios that improve motivation, increase retention, enhance creativity, facilitate more flexibility“ [16, p. 5].

\section{EMgage AT THE KEMPTEN UnIVERSITY OF APPLIED SCIENCES}

\section{A. Basic situation}

At the faculty of computer science at Kempten University of Applied Sciences the total number of students in our software engineering courses is about $50-100$ each semester [17]. The software engineering education is placed in the bachelor programs in computer science and business informatics.

\section{B. Pre-evaluation of the prototype}

\section{1) General setup and research method}

We tested eMgage in our labs and pre-evaluated the prototype in order to receive the feedback of potential users and consider their ideas in the development of the application. The pre-evaluation took place in a software engineering practice with computer science students being in the third semester. Each participant $(n=10)$ was asked to follow a defined workflow after they installed the app. 
The workflow contained to create an account, log in with that account, check the profile page, proceed two learnsessions, check the profile page again, then check the leaderboard and the news area. Finally each of them was prompted to send in a question and related answers as a suggestion and finalize the work by logging out. After completing the workflow the participants were asked to answer some questions in an evaluation sheet. The evaluation sheet contained 34 items which where standardized and thematically structured in 5 areas: the usability of eMgage (15 items), additional functionalities (4 items), possible scenarios (10 items) of usage, general opinion on eMgage (3 items), and personal data (2 items). A marked five-point answer scale (Likert scale) was used, so the participants decided whether they confirm the statements "strongly agree", "agree", "disagree", "strongly disagree", "undecided". In addition each area contained free space for open comments from the participants. The evaluation sheets where processed with EvaSys to gather the results of this pre-evaluation.

\section{2) Results}

The results revealed that the participants were confident with the usability of the prototype. For example they $80 \%$ strongly agreed that eMgage reacts fast on user input. This was one of our basic quality attributes when determining the requirements. Also $80 \%$ of the participants strongly agreed that the depth of the feature structure is adequate which positively influences the search time for certain features. Only the text size was seen as too small by $50 \%$ of the participants, a fact that can be changed quite easily. The free text comments confirmed the results in this area and did not come up with new findings.

In the area additional functionalities the participants were discordant about the configuration possibilities the app should offer. Exemplary the statement "the background color should be individually adjustable" was strongly agreed by $20 \%$, agreed by $20 \%$, disagreed by $30 \%$ and strongly disagreed by $20 \%$ while one participant was undecided. This variance also appears in the results of the other items in this area. This could be explained that the personal taste and preferences could differ a lot from student to student. Three participants stated in the free text comments that there could be a button to log out from the application which should be placed in the header next to the eMgage logo (see Fig. 2). This would reduce the click amount from currently two clicks to one single click.

The following area contained items related to possible scenarios of usage. Fortunately almost all participants were in favor of the idea to use eMgage at home $(80 \%$ strongly agreed), during waiting or travel time $(50 \%$ strongly agreed, $30 \%$ agreed) or directly in university courses (50\% strongly agreed, 30\% agreed). Even $90 \%$ percent strongly agreed or agreed using eMgage for exam preparation while also $90 \%$ strongly agreed that the app supports their personal learning process. One participant argued that "eMgage helps a lot for exam preparation but does not replace it since software engineering is not only about plain factual knowledge".

The next area was about the general opinion on eMgage. For example it was asked if one would recommend eMgage to other students which was answered by $100 \%$ of the participants strongly positive. Also the statement "I would be appreciated to apply eMgage in software engineering education" was strongly agreed by $60 \%$, and agreed by $30 \%$ while one participant was undecided. Two participants argued that it would be better to have repetitive learnsessions. This would mean that learnsessions could be processed how often a user wants to. Actually this thought was one we had at the beginning of the project. We decided not to offer this repetitive structure in the first place since we want to increase the willingness to compete with each other by comparing gained experience points. As the significance of this feature was shown through these comments one could think about a solution which has two different modes: training in which the learnsessions are repeatable and competition.

In general this pre-evaluation confirmed our idea of eMgage and showed that we are on track. We also gained valuable feedback and suggestions for future improvements although the number of participants was quite small and not representative. That is why an evaluation has to follow these findings once the implementation and testing phases of the learning system are completed.

\section{SUMMARY}

In this contribution we presented a concept for a mobile game-based learning system. We described the features of eMgage and their realization with game mechanics and furthermore the relation between them. Our previous findings in increasing the learning motivation of students were adopted in the mobile context. Possible areas of motivational impact and their limitations were shown and discussed. Hence we presented the current state and a preevaluation of the application in one of our software engineering courses.

\section{ACKNOWLEDGMENT}

The authors would like to thank all the participants of the pre-evaluation for their time and the constructive statements.

\section{REFERENCES}

[1] M. Amberg and M. Lang, Innovation durch Smartphone und Co. Die neuen Geschäftspotenziale mobiler Endgeräte. Düsseldorf: Symposium Publishing GmbH, 2011.

[2] P. Figas, G. Hagel, and A. Bartel, The Furtherance of Motivation in the Context of Teaching Software Engineering, in IEEE EDUCON - Special Track "Technical Didactic Software Engineering" (TDSE), Berlin: IEEE Computer Society, 2013.

[3] G. Coulouris, J. Dollimore, T. Kindberg, Verteilte Systeme Konzepte und Design, München: Pearson Studium, 2002.

[4] F. Buschmann, R. Meunier, H. Rohnert, P. Sommerlad, M. Stal, Pattern-Oriented Software Architecture, Volume 1, A System of Patterns, Chichester: Wiley, 1996.

[5] G. Watson, ORMLite [online]. Available: http://ormlite.com [June $25,2014]$

[6] G. Zichermann and C. Cunningham, Gamifcation by Design: Implementing Game Mechanics in Web and Mobile Apps. Sebastopol: O'Reilly Media, 2011.

[7] G. M. Novak, E.T. Patterson, A.D. Gavrin and W. Christian, Justin-Time Teaching: Blending Active Learning with Web Technology, Upper Saddle River, NJ: Prentice-Hall, 1999.

[8] G. Hagel, J. Mottok and M. Müller-Amthor, Drei FeedbackZyklen in der Software Engineering-Ausbildung durch erweitertes Just-in-Time-Teaching, in Software Engineering im Unterricht der Hochschulen (SEUH), 2013, pp. 17-28.

[9] K. Kapp, The Gamification of Learning and Instruction: Gamebased Methods and Strategies for Training and Education, San Francisco: Wiley, 2012.

[10] L. Alfano, M. Horowitch and N. Kitchen. (2010). Cell Phone Use and Concentration During Class [online]. Available: 


\section{SHORT PAPER}

\section{Engaging Students With A MobiLe GAME-BASEd LEARNING SySTEM IN UNIVERSity EdUCATION}

http://www.unh.edu/news/docs/2010CellphoneUsageStudy.pdf [June 25, 2014].

[11] H. Roth, Pädagogische Psychologie des Lehrens und Lernens, Hannover: Schroedel, 1983.

[12] J. Breuer, Spielend lernen? Eine Bestandsaufnahme zum (Digital) Game-Based Learning, in LfM-Dokumentation, vol. 41. Düsseldorf: Landesanstalt für Medien Nordrhein-Westfalen, 2010.

[13] P. Figas, M. Müller-Amthor, A. Bartel, A. Baumgärtner and G. Hagel, Man wächst mit seinen Aufgaben - Über die kompetenzorientierte Konstruktion von Lernaufgaben in der Hochschullehre am Beispiel von Software Engineering, in GfD Fachtagung, Dortmund: Gesellschaft für Fachdidaktik e.V., 2013, in press.

[14] F. Rheinberg, Verhaltenslenkung in Lehr- und Erziehungssituationen, in Motivationsförderung im Schulalltag. Psychologische Grundlagen und praktische Durchführung, F. Rheinberg and S. Krug, Eds. Göttingen: Hogrefe, 2005, pp. 15-23.

[15] A. Bartel, P. Figas and G. Hagel, Mobile Game-Based Learning in University Education. in 21 st Century Education: Constructing meaning and building knowledge in technology supported learning environments, S. Feller and I. Yengin, Eds. Benjamins, 2014, in press.

[16] C.D. Kloos, C. Fernández-Panadero, B. Ibáñez; M. Muñoz and A. Pardo, M-learning will Disrupt Educational Practices, in IEEE EDUCON, Marrakesh: IEEE Computer Society, 2012.

[17] J. Abke et al., EVELIN. Ein Forschungsprojekt zur systematischen Verbesserung des Lernens von Software Engineering, in Tagungsband ESE-Kongress, Sindelfingen: Elektronikpraxis, 2012, pp. 653-658.

\section{AUTHORS}

A. Bartel and G. Hagel are with the Faculty Computer Science, Kempten University of Applied Sciences, Germany (\{alexander.bartel, georg.hagel\}@hs-kempten.de).

This work is part of the EVELIN project which is supported by the Bundesministerium für Bildung und Forschung with the project number 01PL12022C. Submitted 30 June 2014. Published as resubmitted 14 October 2014. 